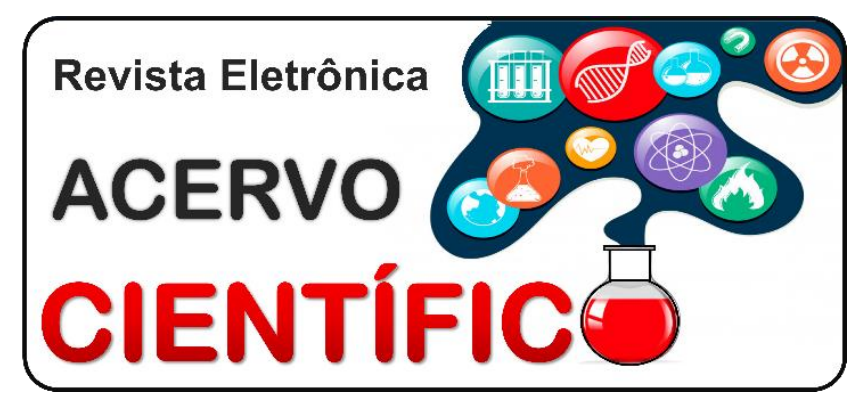

ARTIGO ORIGINAL

Recebido em: 6/2020

Aceito em: 7/2020

Publicado em: 12/2020

\title{
Saúde mental: visão e atitudes dos formandos de medicina
}

\author{
Mental health: vision and atitudes of medical trainers
}

Salud mental: visión y actitudes de entrenadores de medicina

Tanise Nazaré Maia Costa ${ }^{1}$, Bruna Letícia Kzan Pedrosa ${ }^{1 *}$, Amanda Vallinoto Silva de Araújo,

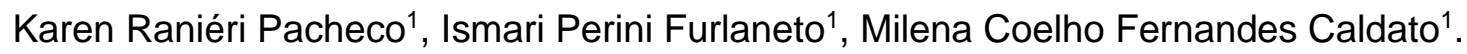

Resumo: Avaliar as condutas dos concluintes do curso de medicina em relação a pacientes psiquiátricos. Trata-se de um estudo transversal, descritivo e analítico, no qual um questionário estruturado foi aplicado a acadêmicos de medicina do sexto semestre. Foram avaliados 111 estudantes concluintes do curso de medicina, sendo $55,9 \%$ do sexo feminino e $44,1 \%$ do sexo masculino, a idade média foi de 25,3 anos. No estudo não houve diferença significativa na proporção das diferentes respostas as perguntas a respeito da hospitalização do paciente psiquiátrico; nos itens que se referem a aptidão dos acadêmicos em assistir um paciente doente mental, notou-se baixa frequência de respostas "concordo totalmente" quando comparadas às demais. A maioria dos discentes optou por responder "discordo totalmente" ao item que se refere a atribuição do atendimento dos pacientes psiquiátricos e, quanto ao item que avalia a capacidade individual do paciente em tomar decisões, a maioria das respostas se concentrou em "tenho dúvidas" e "discordo em parte". Entre os acadêmicos, há a ciência da necessidade de uma melhor assistência ao paciente psiquiátrico, por meio do aconselhamento dos responsáveis por formar o ambiente social do doente mental.

Palavras-chave: Saúde mental, Educação médica, Estudantes de medicina.

Abstract: Evaluate how the medical school graduates are conducting towards psychiatric patients. This is a cross-sectional, descriptive and analytical study, in which a structured questionnaire was applied to medical students in the sixth semester. 111 graduating medical students were evaluated, $55.9 \%$ female and $44.1 \%$ male, the average age was 25.3 years. In the study, there was no lower difference in the proportion of different responses to questions regarding the hospitalization of psychiatric patients; in the items that serve the students' aptitude to assist a mentally ill patient, there was a low frequency of "totally agree" responses when compared to the others. Most students chose to answer "totally disagree" to the item that refers to the care of psychiatric patients and, to the item that assesses the patient's individual ability to make decisions, most of the answers focused on "I have doubts" and "partly disagree". Among academics, there is a knowledge of the need for better assistance to psychiatric patients, through the counseling of those responsible for forming the social environment of the mentally ill.

Keywords: Mental health, Education medical, Students medical.

\footnotetext{
${ }^{1}$ Centro Universitário do Pará (CESUPA), Belém - Pará.

*E-mail: bruhkzan@gmail.com
} 
Resumen: Evaluar cómo los graduados de la escuela de medicina se están comportando con los pacientes psiquiátricos. Se trata de un estudio transversal, descriptivo y analítico, en el que se aplicó un cuestionario estructurado a estudiantes de medicina del sexto semestre. Se evaluaron 111 estudiantes de medicina egresados, $55,9 \%$ mujeres y $44,1 \%$ hombres, la edad promedio fue de 25,3 años. En el estudio, no hubo menor diferencia en la proporción de diferentes respuestas a las preguntas sobre la hospitalización de pacientes psiquiátricos; en los ítems que sirven a la aptitud de los estudiantes para asistir a un enfermo mental, hubo una baja frecuencia de respuestas "totalmente de acuerdo" en comparación con los demás. La mayoría de los estudiantes optaron por responder "totalmente en desacuerdo" al ítem que se refiere a la atención al paciente psiquiátrico y, al ítem que evalúa la capacidad individual del paciente para tomar decisiones, la mayoría de las respuestas centrado en "tengo dudas" y "en parte en desacuerdo". Entre los académicos, existe un conocimiento de la necesidad de una mejor asistencia a los pacientes psiquiátricos, a través del asesoramiento de los responsables de formar el entorno social de los enfermos mentales.

Palabras clave: Salud mental, Educación medica, Estudiantes de medicina.

\section{INTRODUÇÃO}

A assistência psiquiátrica, durante muitos séculos, sofreu modificações de acordo com a percepção da sociedade a respeito das necessidades dos doentes, evoluindo conceitos e valores até o estágio de cuidado e atendimentos realizados atualmente. A partir da Segunda Grande Guerra Mundial, com os avanços econômicos e sociais, movimentos civis, profissionais e culturais começaram a se desenvolver e a compreensão a respeito do auxílio aos doentes mentais sofreu alterações, reformulando os princípios dos hospitais psiquiátricos. Desde então, com os avanços alcançados após a Segunda Guerra Mundial e a criação de novas organizações, como a Organização Mundial de Saúde (OMS), o conceito de assistência psiquiátrica evoluiu mundialmente, promovendo reformas especificas em cada país (MACIEL SC, 2012).

A reforma psiquiátrica, no Brasil, teve seu início nos anos de 1970, após denúncias sobre maus-tratos e desassistências divulgados pela mídia, além da ebulição de debates político-filosóficos a respeito dos direitos humanos dos pacientes psiquiátricos. Teve como objetivo principal questionar os princípios que regiam a internação do doente mental nos hospitais psiquiátricos e os moldes da prática médica ao assistir esses indivíduos, aceitando não mais o conceito de isolamento e segregação desses pacientes, mas sim reabilitação ativa e assistência humanizada multiprofissional (MACIEL SC, 2012).

Com o decorrer dos anos a assistência psiquiátrica passou por muitas modernizações e avanços, até que em 2017 foi publicada, pelo Ministério da Saúde, a portaria oㅡ 3.588, que tem como objetivo redirecionar a política de assistência ao doente mental. A importância deste documento consiste em desatar preconceitos direcionados aos pacientes psiquiátricos que foram se alastrando durante os últimos 30 anos. Esses retrocessos foram liderados por uma militância ideológica de profissionais não médicos que ocupou a coordenação de saúde mental do Ministério da Saúde. Eles acreditavam que o doente mental não é doente e sim diferente, e que não precisava de um acompanhamento médico e leitos especializados (BRASIL, 2017).

Atualmente, um dos pontos chaves ao auxílio psiquiátrico é a necessidade de uma equipe interdisciplinar, que conta com um médico, um enfermeiro, um psicólogo e um terapeuta ocupacional. A formação dessa equipe tem como objetivo a fragmentação do conhecimento e a desconstrução da hierarquia e dos limites técnicos de cada integrante. Dessa forma, a compreensão dobre as necessidades dos pacientes psiquiátricos tornou-se mais ampla, estabelecendo a noção de multiplicidade dos problemas e importância em agregar múltiplas terapias, além de inserir os familiares como um dos pilares para uma assistência efetiva ao doente (MACIEL SC, 2012).

Após a desinstitucionalização psiquiátrica e a consolidação de um conhecimento mais amplo acerca da saúde mental, a integração de profissionais de múltiplas especialidades contribuiu para o esclarecimento e compreensão mais abrangente do quadro do paciente psiquiátrico, sendo assim constituída por: psiquiatra (coordenador), clínico, enfermeiro, assistente social, psicólogo, farmacêutico, nutricionista, terapeuta 
ocupacional, educador físico e profissionais de nível médio. Dentro desse cenário, a prática médica começou a se desenvolver e a evoluir ao adotar métodos inovadores de cuidado e assistência aos pacientes psiquiátricos, assegurando o diagnóstico, a classificação e a forma terapêutica a ser abordada nos transtornos mentais e comportamentais (SOUSA PF, et al, 2016).

A vertente do ensino, em particular, tem a necessidade de mudança no quesito ensino em saúde mental, com o objetivo de melhorar a prática do atendimento. Ainda que as diretrizes curriculares nacionais do curso de graduação em medicina garantam que todo acadêmico de medicina deva reconhecer a saúde como direito e atuar de forma a garantir a integralidade da assistência entendida como conjunto articulado e continuo de ações e serviços preventivos e curativos, individuais e coletivos, exigidos para cada caso em todos os níveis de complexidade do sistema, alguns estudos sustentam que uma das necessidades é a melhor articulação entre prática e teoria de forma que os futuros médicos possam aprender sobre as relações interpessoais, produção do cuidado, solidariedade e acolhimento, tendo, acima de tudo, criticidade sobre o seu papel dentro do amparo ao paciente psiquiátrico (VILLELA SC e SCATENA MC, 2004).

Após um longo período do predomínio desse velho paradigma acerca da introdução do acadêmico de medicina na assistência ao paciente psiquiátrico, algumas universidades brasileiras, como a Universidade de Londrina, têm despertado para esta temática, modificando a metodologia de ensino de maneira que englobe as necessidades do discente em atender um indivíduo com transtorno mental. Desta forma, foi aplicado um eixo metodológico que dá destaque a Aprendizagem Baseada em Problemas (ABP), que a partir de uma situação-problema, busca-se resolução do caso, por meio de dados, que são analisados e discutidos; e finalmente, são elaboradas hipóteses de solução, que devem ser testadas e comprovadas. A partir disso, o discente consegue relacionar as áreas do conhecimento, habilidades e atitudes relacionadas a prática da relação médico-paciente (NUNES SO, et al., 2008).

É importante a produção desse estudo pois, apesar da dificuldade em se encontrar parâmetros para comparar os perfis de estudantes de medicina de diferentes universidades, acredita-se que o conhecimento sobre doença mental possa servir de modelo de comparação, já que esse se configura como fator independente das grades curriculares dentre as universidades. Com isso, independentemente das propostas curriculares universitárias, como carga horaria de aulas, estágios, projetos, o discente de medicina deve apresentar uma atitude humanizada em relação a pacientes psiquiátricos, de acordo com os princípios da reforma psiquiátrica brasileira (AMARANT P, 2008).

Os futuros médicos, portanto, precisam estar preparados para atender os pacientes com limitações e seus familiares, visando apoiá-los e tratá-los de maneira que valorize não apenas a doença, mas sim a pessoa de forma integral, reinserindo os pacientes ao convívio social com medidas qualificadas, as quais foram aprendidas e construídas durante o processo de formação acadêmica.

O objetivo do presente artigo é avaliar a atitude dos concluintes do curso de Medicina sobre aspectos relacionados a saúde mental e apontar dificuldades relacionados ao tema saúde mental que possam servir como subsídios para elaborar material de apoio para desenvolvimento em competências em habilidades humanísticas no curso de Medicina.

\section{MÉTODOS}

Trata-se de um estudo transversal, descritivo e analítico, realizado por meio de questionário previamente estruturado, aplicado a estudantes concluintes do sexto ano do curso de Medicina de duas instituições do Estado do Pará, sendo uma privada e outra pública.

O período de coleta do estudo foi de agosto a novembro de 2016. Participaram do estudo os alunos do sexto ano do curso de Medicina. Para estar presente no estudo, foram incluídos os alunos que estavam matriculados no sexto ano do curso de Medicina de duas instituições escolhidas e aceitaram a participar do assunto e assinaram o Termo de Consentimento Livre e Esclarecido (TCLE). A coleta dos dados foi realizada pela aplicação de um questionário do tipo "Likert" validado e intitulado Escala de Atitudes Frente a 
Algumas Questões da Prática Médica, elaborado na Faculdade de Medicina de Ribeirão Preto da Universidade do Estado de São Paulo (COLARES et al, 2002).

Possui cinquenta e dois itens indagando sobre diversas situações médicas e de bioética. Esta escala foi aplicada para a confecção da tese "Percepção dos concluintes de Medicina sobre aspectos relacionados a terminalidade da vida", com a utilização de apenas cinco perguntas. Porém, com objetivo de não desprezar as outras informações, foram criadas extensões do estudo fazendo uso de sete questionamentos concernentes sobre a pesquisa médica.

Os dados foram submetidos ao teste estatístico de teste de Qui-quadrado de aderência pelo programa Bio-Estat 5. A pesquisa foi aceita no Comitê de Ética em Pesquisa (CEP) com seres humanos sob CAAE 54831916.8.0000.5168 e número de parecer 1.580.371.

Os alunos participaram da presente pesquisa apenas após esclarecimento a respeito dos objetivos e assinatura do TCLE, concordante com os termos da Resolução 466/12 do Conselho Nacional de Saúde do Ministério da Saúde.

\section{RESULTADOS}

Participaram do estudo 111 estudantes concluintes do curso de medicina, sendo $55,9 \%$ do sexo feminino (62/111; IC95\% 46,6 - 64,7) e 44,1\% do sexo masculino (49/111; IC95\% 35,3 - 53,4). A idade média dos participantes foi igual a 25,3 anos $\pm 4,0$ anos, variando entre 22 e 52 anos (IC95\% 24,6-26,1).

A Figura 1 apresenta a distribuição dos respondentes quanto à concordância com os itens do questionário que apresentavam juízo negativo no que diz respeito aos sentimentos do estudante de medicina diante do doente mental e dificuldades enfrentadas, além de aspectos relacionados às políticas de saúde específicas na área de saúde mental.

Não foi observada diferença significativa na proporção das diferentes respostas aos itens 3 e 7 ( $p>0,05)$; quanto aos itens 1 ("Sinto-me incomodado em atender pacientes com sinais sugestivos de problemas psiquiátricos"), 2 ("Os pacientes psiquiátricos são os que mais me incomodam durante uma consulta") e 5 ("O paciente com doença mental me desperta sentimentos negativos"), notou-se baixa frequência de respostas "concordo totalmente" quando comparadas às demais opções $(5,4 \%, p<0,01 ; 8,2 \%, p<0,05$; $2,7 \%, p<0,01$, respectivamente).

A maioria significativa dos discentes optou por responder "discordo totalmente" ao item $4(60,4 \%$, $p<0,01$ ) e, quanto ao item 6 ("Penso que os doentes mentais crônicos não tem condições de tomar decisões sobre sua própria vida"), a maioria das respostas se concentrou em "tenho dúvidas" e "discordo em parte" $(27,9 \%$ e $30,6 \%, p<0,01$, respectivamente) (Figura 1).

No que diz respeito aos itens que dissertavam positivamente sobre os aspectos relacionados à doença mental, a maioria significativa dos estudantes respondeu estar "totalmente de acordo" aos os itens 2 ("Acho que o médico pode ser um profissional de ajuda para o doente mental, sem ser necessariamente um psicoterapeuta"), 3 ("Acredito na importância dos fatores do ambiente social na evolução da doença mental"), 4 ("Considero importante conhecer as políticas atuais na área da saúde mental mesmo não atuando na área") e 5 ("Tenho facilidade para conduzir a entrevista com pacientes psiquiátricos"), variando de $59,5 \%$ (item 2) a 75,2\% (item 3) ( $p<0,01)$ (Figura 2 ).

Quanto ao item 1 ("Penso que as políticas de socialização do doente mental podem amenizar seus problemas"), observou-se tendência de maior frequência de respostas às opções "tenho dúvidas" e "discordo em parte" (27,0\% e 27,9\%, p<0,01, respectivamente) (Figura 2). 
Figura 1 - Distribuição dos estudantes quanto a concordância com os itens de sentido negativo no contexto do paciente com doença mental e suas dificuldades e das políticas públicas específicas para essa população.

\section{Sinto-me incomodado em atender pacientes com sinais sugestivos de problemas psiquiátricos.***}

2. Os pacientes psiquiátricos são os que mais me incomodam durante uma consulta.**

3. Acho que um paciente psiquiátrico, com problemas orgânicos, não deve ser internado em enfermaria geral.*

4. Considero que a investigação de aspectos psicológicos envolvidos nas doenças cabe somente aos profissionais da área de...

5. O paciente com doença mental me desperta sentimentos negativos. ${ }^{* *}$

6. Penso que os doentes mentais crônicos não têm condições de tomar decisões sobre sua própria vida.***

7. Penso que me sentiria incomodado atuando em Hospital Psiquiátrico.*

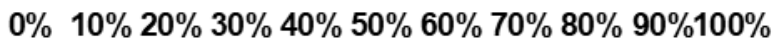
Frequência

$\square$ Concordo totalmente

$\square$ Concordo em parte

$\square$ Tenho dúvidas

Legenda: ${ }^{* *} p<0,01 ;{ }^{* *} p<0,05 ;{ }^{*} p>0,05$ (Qui-Quadrado de aderência).

Fonte: COSTA TNM, et al., 2020.

Figura 2 - Distribuição dos estudantes quanto a concordância com os itens de sentido positivo no contexto do paciente com doença mental e suas dificuldades e das políticas públicas específicas para essa população.

1. Penso que as políticas de socialização do doente mental podem amenizar seus problemas.***

2. Acho que o médico pode ser um profissional de ajuda para o doente mental, sem ser necessariamente um psicoterapeuta.***

3. Acredito na importância dos fatores do ambiente social na evolução da doença mental.***

4. Considero importante conhecer as políticas atuais na área de saúde mental mesmo não atuando na área.***

5. Tenho facilidade para conduzir a entrevista com pacientes psiquiátricos.***

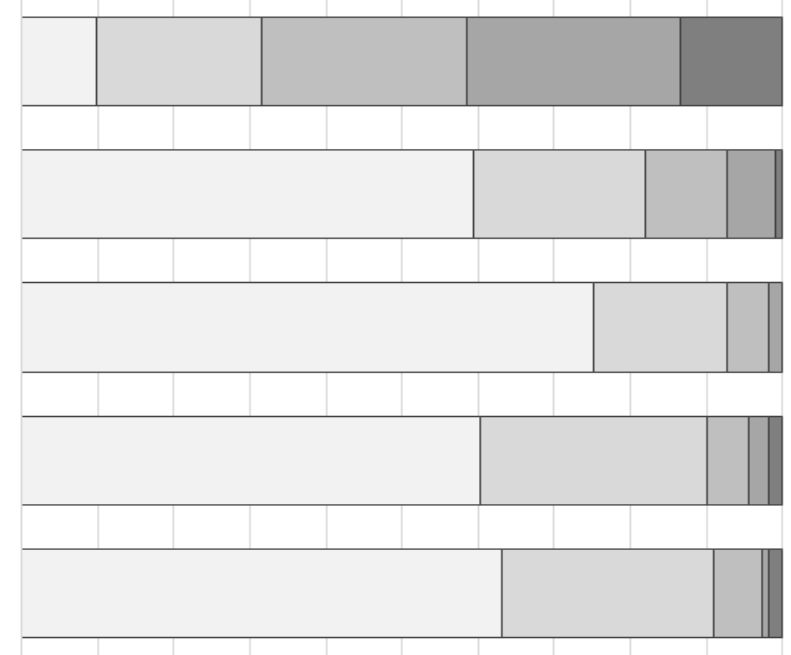

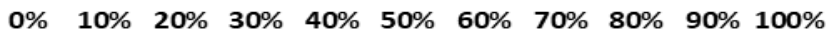
Frequência

$\square$ Concordo totalmente $\square$ Concordo em parte $\square$ Tenho dúvidas $\square$ Discodo em parte $\square$ Discordo totalmente

Legenda: ${ }^{* * *} p<0,01$ (Qui-Quadrado de aderência). Fonte: COSTA TNM, et al., 2020. 


\section{DISCUSSÃO}

As mudanças ocorridas ao longo dos séculos acerca do saber psiquiátrico foram de extrema importância para o desenvolvimento de uma assistência mais integral e humanizada ao paciente psiquiátrico, tendo em vista que muitas modificações carecem ainda de implementação efetiva. Portanto, é importante observar e analisar a efetividade e prática das ações instituídas atualmente, tanto nos centros de referência psiquiátricos quanto nas instituições de ensino superior, em busca de uma preparação profissional adequada para assistir a esses indivíduos. A partir disso, esse estudo busca avaliar a preparação acadêmica dos estudantes de medicina em relação ao atendimento psiquiátrico, evidenciando as principais carências que ainda persistem nas metodologias de ensino-aprendizado adotadas pelas instituições atualmente (SANTOS SS, 2013).

Ainda que muitos discentes tenham indicado certa facilidade em atender pacientes psiquiátricos, nos itens de sentido positivo presentes na Figura 2, é possível inferir que permanece um certo desconforto em assistir um doente mental de acordo com os resultados dos itens da Figura 1. Diante do exposto, faz-se o questionamento: a "facilidade" em atender o paciente psiquiátrico significa uma assistência eficaz e dentro dos preceitos implementados pela reforma?

Os fatores que consolidam esta discussão podem ser atribuídos à capacitação básica insuficiente nos centros universitários durante a formação acadêmica, à falta de interesse dos acadêmicos em aprofundar o conhecimento na área da saúde mental, à seletividade pela especialização que o estudante almeja futuramente ou, até mesmo, à perseverança de velhos paradigmas e preconceitos a respeito de pessoas com transtorno mental. Esse contexto dificuldade a integralidade do atendimento desses pacientes haja vista com mais médicos com o desconforto de atender pacientes psiquiátricos e muitos se negando a atendê-los, aumenta a quantidade de pacientes por médico, aumentando filas, dificultando conseguir consultas e um tratamento regular (MACIEL SC, 2007).

Mesmo diante das mudanças implementadas na graduação em medicina, ainda se observa muita relutância a essa modificação, privilegiando aspectos deterministas, hospitalocêntricos e individualista. Esse contexto gera um impacto na relação saúde-doença do paciente psiquiátrico que encontrava atendimento exclusivamente em hospitais ou centros especialistas, dessa forma, a atenção básica construiu uma pavimentação importante para diminuição dessa realidade ao incluir o Programa de Atenção ao Paciente Psiquiátrico como um dos programas que devem funcionar nas unidades básicas de saúde com atendimento geral, dispensação de medicamentos específicos, atenção à saúde e educação em saúde feita pelos profissionais médicos, enfermeiros e agentes comunitários de saúde integrantes da atenção básica, contudo a integralidade do atendimento ao paciente esbarra nos problemas educacionais e técnicos que aparecem durante a graduação médica e não são sanados (NOGUEIRA MI, 2009).

Tal assertiva também foi abordada em uma pesquisa realizada por Pagliosa FL (2006), com acadêmicos de medicina da Universidade Federal de Santa Catarina, a qual revelou que o ensino permanece pautado na aprendizagem de técnicas e desenvolvimento de competências teóricas, possuindo um elevado entendimento do processo saúde-doença, com forte enfoque biológico. Além disso, percebeu-se grande preocupação acerca da compreensão do funcionamento do corpo humano, dando destaque para disciplinas como anatomia e desmerecendo e desconsiderando disciplinas que não apresentavam caráter biológico, como Antropologia e sociologia. Esse contexto pode gerar consequências graves como a diminuição do desenvolvimento de aspectos humanísticos e antropológicos pelos graduandos de medicina que estão inseridos nos cursos de medicina tecnicistas, diminuindo a quantidade de profissionais médicos no futuro preocupados com o bem estar biopsicossocial do paciente, mas sim com focados na doença e em seu tratamento. Dessa forma, observa-se que os universitários possuem certa diferença no processo de assimilação dos preceitos da reforma psiquiátrica, sendo ainda um sistema em construção, pautado no velho paradigma da necessidade de internação desses pacientes em instituições psiquiátricas.

Concomitantemente, percebe-se que quando a saúde mental é abordada na prática médica, o discente vê-se com dificuldades para relacionar-se com o paciente e para proporcionar uma assistência satisfatória, como prediz a reforma, prejudicando, assim, a construção de um atendimento humanizado e eficaz. Nesse 
sentido, entender que o paciente psiquiátrico é destinado a instituições psiquiátricas, nega a possibilidade de atender na atenção básica onde há o Programa de Atenção ao Paciente Psiquiátrico com desenvolvimento do bem estar social e familiar do paciente com esta patologia. Dessa forma, é prioritário que ocorram mudanças em aspectos durante a graduação médica a fim de diminuir o preconceito, aumentar a humanização e melhore $o$ atendimento de pessoas com doenças psiquiátricas (BORGES RF e LUZIO CA, 2010).

Os resultados da má qualificação profissional do acadêmico de medicina durante o curso impactam na atuação tanto deles quanto dos médicos, dificultando a formação de uma equipe interdisciplinar adequada e favorecendo a superlotação de centros especializados no cuidado de pacientes psiquiátricos. Essa realidade, além de superlotar os centros especializados, diminui a possibilidade de um atendimento integral haja vista pela quantidade de pacientes, diminuir o tempo de consulta e focando apenas na doença.

Ainda que os resultados revelem certo desconforto dos acadêmicos de medicina quanto aos itens de sentido negativo no contexto do paciente com saúde mental, os discentes demonstraram boa compreensão acerca da necessidade da formulação de políticas públicas e a importância do meio social em que reside o paciente, promovendo o aprimoramento e aperfeiçoamento da reforma psiquiátrica. Dessa forma, os pacientes conseguirão ser atendidos com maior integralidade pois haverá mais profissionais médicos discutindo desde a graduação, melhorando a humanização e diminuindo o preconceito de atender pacientes psiquiátricos.

\section{CONCLUSÃO}

O objetivo deste estudo foi avaliar e analisar a atuação do estudante de medicina na área da saúde mental, considerando as suas dificuldades e obstáculos que impactam na implementação plena da reforma psiquiátrica no Brasil. Os achados desta pesquisa indicaram, entre os acadêmicos, a ciência da necessidade de uma melhor assistência ao paciente psiquiátrico, por meio da compreensão e aconselhamento dos responsáveis por formar o ambiente social do doente mental, além da importância da formulação de novas políticas públicas direcionadas a esse grupo de indivíduos. Contudo, nota-se ainda certo impasse entre os discentes com relação a capacidade de fazer um atendimento e assistência eficaz ao paciente com transtorno mental. É coerente assumir que a visão negativa do doente mental, marcada por termos pejorativos e estereótipos, e a falta de qualificação adequada representam um dos grandes desafios para socialização do portador de transtorno mental e instituição plena da reforma psiquiátrica. Isso exigirá tempo e dedicação dos futuros profissionais da saúde em superar esses obstáculos.

\section{REFERÊNCIAS}

1. AMARANTE P. Saúde Mental e atenção psicossocial. Editora Fiocruz [Internet]. 2008; (4ª edição).

2. MACIEL SC. Reforma psiquiátrica no Brasil: algumas reflexões. Cad. Bras. Saúde Mental, Rio de Janeiro, 2012; 4(8), 73-82.

3. MACIEL SC. Exclusão/inclusão social do doente mental/louco: representações e práticas no contexto da reforma psiquiátrica [tese na Internet]. Universidade Federal da Paraíba; 2007.

4. NOGUEIRA MI. As mudanças na educação médica brasileira em perspectiva: reflexões sobre a emergência de um novo estilo de pensamento. Rev. bras. educ. med, 2009, 33(2): 262-270.

5. PAGLIOSA FL. Percepções sobre saúde e doença dos estudantes da primeira fase do Curso de Medicina da UFSC [dissertação mestrado on the Internet]. Universidade Federal de Santa Catarina; 2006.

6. BORGES RF, LUZIO CA. Pesquisa qualitativa em saúde mental: alguns apontamentos. Rev. Psicol [Internet]. 2010, 9(1): 14-23.

7. VILLELA SC, SCATENA MC. A enfermagem e o cuidado na área de saúde mental. Rev. bras. enferm [Internet]. 2004, 57(6): 738-741.

8. NUNES SO, et al. O ensino de psiquiatria, habilidades de comunicação e atitudes no currículo integrado do curso de Medicina da Universidade Estadual de Londrina. Rev. bras. educ. med [Internet]. 2008, 32(2): 210-216.

9. SANTOS SS, et al. Atitudes, conhecimento e opinião frente à saúde mental em alunos de graduação em enfermagem. Rev. Esc. Enferm. USP 2013; 47(5):1202-10.

10. SOUSA PF, et al. Atitudes e Representações em Saúde Mental: Um Estudo com Universitários. Psico-USF, 2016; 21(3). 
11. BRASIL. Ministério da Saúde. Nova política nacional de saúde mental. Portaria no 3.588 [internet]. 21 de dezembro de 2017.

12. BRASIL. Ministério da Educação. Conselho Nacional de Educação. Câmara de Educação Superior. Resolução CNE/CES no 3, de 20 de junho de 2014. Institui diretrizes curriculares nacionais do curso de graduação em Medicina. Diário Oficial da União. Brasília, 2014; seção 1: 8-11.

13. CFM, 2018. In: Assistência ao doente mental no rumo certo. Disponível em: https://portal.cfm.org.br/index.php?option=com_content\&view=article\&id=27454. Acesso em: 25 ago. 2020.

14. DIRETRIZES DO PROJETO POLÍTICO-PEDAGÓGICO [Internet]. Londrina: Universidade Estadual de Londrina; 2004. Colegiado do Curso de Medicina. 